\title{
HYBRID APPROACH TO SOLVE THE PROBLEM OF PAPR IN OFDM SIGNAL: A SURVEY
}

\author{
Ravindra Bankapur ${ }^{1}$, Mallikarjun.N.Deshmukh², Ambika Haranal ${ }^{3}$, T.Y.Melligeri ${ }^{4}$ \\ ${ }^{I}$ P.G. Scholar Department of ECE, B.L.D.E.A's Dr. P.G.Halakatti College of Engineering and Technology Vijayapur, \\ Karnataka, India - 586103 \\ ravindrabankapur@gmail.com \\ ${ }^{2}$ Associate Professor Department of ECE, B.L.D.E.A's Dr. P.G.Halakatti College of Engineering and Technology \\ Vijayapur, Karnataka, India - 586103 \\ mndeshmukh@yahoo.com \\ ${ }^{3}$ PG Scholar Dept. of ECE, B.L.D.E.A's Dr. P.G.Halakatti College of Engineering and Technology Vijayapur-586103, \\ Karnataka, India - 586103 \\ ambikaharanal@gmail.com \\ ${ }^{4}$ Assistant Professor Department of ECE, B.L.D.E.A's Dr. P.G.Halakatti College of Engineering and Technology \\ Vijayapur-586103, Karnataka, India - 586103 \\ melligeri2005@yahoo.com
}

\begin{abstract}
"Orthogonal frequency division multiplexing(OFDM)" is one of the most promising digital modulation come multiple access method used to achieve the today's need of wireless communication specially used for providing $4 g$ plus services developed by $3 G P P$ Association for the use of LTE. The OFDM has many advantageous characteristics such as higher spectral efficiency, higher data rate, higher bit rate, mitigated delay spread, lower multipath distortion, along with this characteristic features, OFDM system has few major issues associated with the system performance. One of the major issues is" peak to average power ratio (PAPR)" associated with the signal. Researchers have proposed many techniques to minimize the PAPR such as " interleaved OFDM, selective mapping (SLM) and partial transmit sequence(PTS)" under signal scrambling techniques and peak windowing, clipping, filtering, tone rejection(TR), tone injection(TI), companding under signal distortion techniques. Many coding techniques such as block coding or pre-coding, Hadamard code hamming code, Reed Solomon, cyclic codes etc. many of the techniques able to reduce PAPR for some extent but most of them suffers from system complexity and power constraints. In this paper we study about many PAPR reduction techniques along with we will discuss about newly introduced hybrid PAPR reduction techniques which can give better result as compared with earliest.
\end{abstract}

Keywords: 3GPP, PAPR, Signal Scrambling Techniques, Etc

\section{INTRODUCTION}

Increased demand for wireless communication lead to the rapid development in the radio mobile communication. So various multicarrier modulation techniques have been evolved with the aim of reaching the need of wired as well as wireless communication systems, one of is the most promising multiplexing technique is Orthogonal Frequency Division Multiplexing (OFDM) which fulfills the requirements of digital communication providing advantageous characteristics such as higher spectral efficiency, higher data rate, mitigated delay spread, lower multipath distortion, Bandwidth flexibility.

With the multicarrier signals and with introduction of orthogonality among the subcarriers OFDM started ruling out the today's world in communication by taking its position to higher-speed data transmission applications such as Digital Audio Broadcasting (DAB), terrestrial digital video broad casting (DVB-T), the IEEE 802.11a (WLAN),IEEE $802.11 / \mathrm{g} / \mathrm{n}$, IEEE802.16d (WMAN),
Hyperlan, wireless ATM transmission system. The Association of 3rd Generation Partnership Project's Long Term Evolution (3GPP LTE) was the first to adopt OFDM system in downlink transmission.

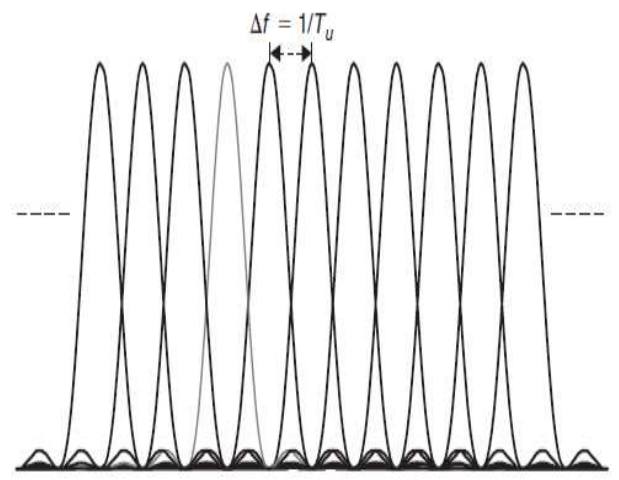

Fig 1. Spectrum of OFDM.

We can see the spectrum of OFDM as depicted in Figure.1 
The fundamental principle of OFDM is that source data is processed and is divided into several parallel streams of channels, one channel for each subcarrier and the orthogonal subcarriers are submitted to synchronization and demodulation is carried out by means of IDFT/IFFT, followed by channel equalization, decoding followed by deinterleaving. Inverse Fast Fourier Transform/Fast Fourier Transform is used to carry out modulation and demodulation of the symbols respectively. The resistance of the system to the errors caused by multipath propagation of signal is determined by the length of the IFFT/FFT. We can see the OFDM system block diagram in Figure. 2

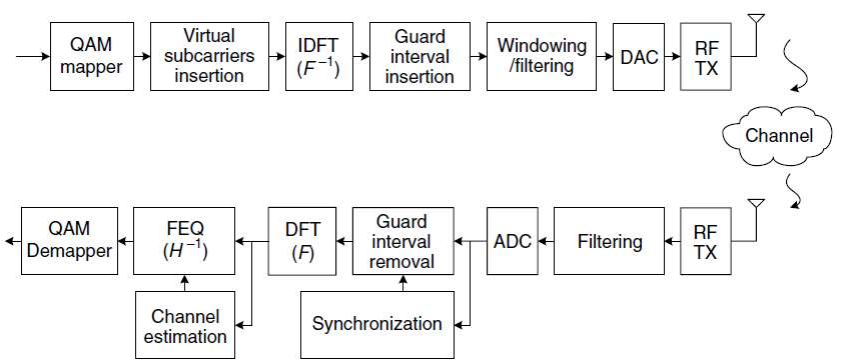

Fig 2. OFDM system block diagram

As the OFDM is a multicarrier scheme it often introduces PAPR casued by different subcarriers with same phase. PAPR is a major issue associated with the system, which drives the power amplifier into non linear region of power amplifier BER degradation which affects the system performance.

In the discussion, we will study diffent types of PAPR reduction techniques which are mainly classified as signal scrambling, signal distortion and coding techniques along with this we study a new hybrid approach is used for PAPR minimization in OFDM signal.

In literature study, we will discusss what is PAPR? What are its effects on the performance of the system in section II, and availalable reduction methods different kinds of "signal scrambling techniques", "signal distortion techniques" and Many "coding techniques" such as block coding or precoding, Hadamard code are studied in detail in section III, the newly found hybrid approach for redcution of OFDM is also discussed under section IV, finally we conclude the the literature study about suitable PAPR reduction technique that given better performance in OFDM system in section $\mathrm{V}$.

\section{THE PAPR IN OFDM SIGNAL}

The PAPR is the reason for reduction in efficiency of Radio-Frequency power amplifier used. Due to formation of complexity in ADCs and DACs.

$$
\operatorname{PAPR}(\mathrm{x})=\frac{\max |\mathrm{x}(\mathrm{t})|^{2}}{\mathrm{E}\left[|\mathrm{x}(\mathrm{t})|^{2}\right]}
$$

Where $E[$.$] denotes the expectation operator.$
PAPR occurs in a multicarrier system when different sub carriers are out of phase with each other, at every instant they are different to each other at different phase values, when all the carriers reaches same value simultaneously then a shoot will occur in the output signal that we call as peak value. The value of peak signal can be very high as compared to the average value of the signal. This ratio of the peak to average power value we term it as peak to average power ration (PAPR). We can see the characteristic curve of HPA as shown in fig 3.

\section{REDUCTION TECHNIQUES OF PAPR}

The PAPR reductions techniques are mainly classified as "signal scrambling, signal distortion" and coding techniques.

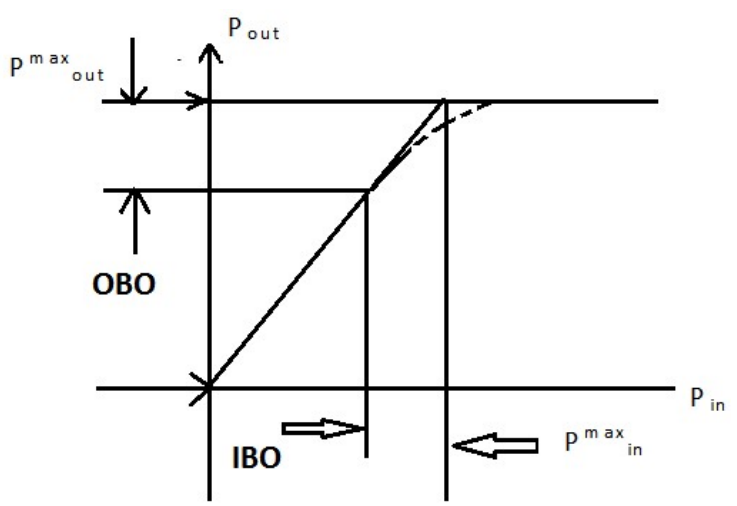

Figure 3. Characteristic curve of HPA

The available PAPR reduction techniques SLM, PTS and interleaved OFDM are under signal scrambling techniques and peak windowing; clipping and "filtering", tone rejection, tone injection, companding under signal distortion techniques. Many coding techniques such as block coding or pre-coding, Hadamard code hamming code, etc

a) Signal scrambling techniques: the most used techniques the basic idea behind this technique is that it select the sequence of signals with least PAPR value the techniques come under this category are SLM,PTS and Interleaved OFDM.

1. Selected Mapping Technique (SLM): It is a part of scrambling technique in which a set of signal are transmitted with representation of some information in each signal. Among the transmitted signals a sequence of signal with very minimum PAPR value is scrambled and transmitted over the channel. The selected signals information must be sent to the receiver along with selected signal as signal side information (SSI) in order to decode the original information at the receiver. Suppose if there is any mistake or error in the sent side information then it is very difficult to receive signal at receiver. The side information to be transmitted should have protection to receiver original signal along with selected signal. Once the receiver has been received this side information then decoding of the transmitted signal becomes easy. Fig. 4 shows the block diagram of SLM technique. 
Even though SLM method uses codes it's been limited for only PAPR reduction and not any error correction codes. If the generated phase sequence is cyclically shifted version of previous sequence. Information needed to transmit will be less.

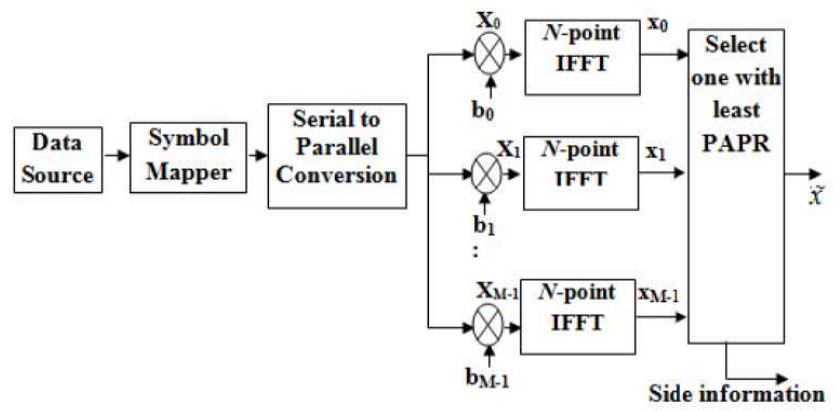

Figure. 4 block diagram of selected mapping

2. Partial transmit sequence (PTS): The PTS technique is most popularly used method used in reduction of PAPR. The concept of PTS Scheme can be understood by looking at its bock diagram this is as depicted in Fig. 5.

The idea behind the PTS scheme is that it divides the original sequences of OFDM into several sequences and multiplies each sequence by distinct weights till the we get the best results. The PTS block Diagram shows that the block of $\mathrm{N}$ symbols input data is portioned into many alternate sub blocks and then information sequences are further transmitted. The portioning of sub-blocks is one another factor, as it also affects the PAPR reduction performance which should be taken into consideration. Sub blocks can be partitioned in three ways as adjacent, interleaved and pseudo-random portioning. Due to use of more number of sub-blocks so this requires more IFFT for computation this drives the system into complexity. Along with this it also adds SII (Side Information Index) as that in SLM scheme. That is also concern for system to take care of SSI that should be sent to receiver for decoding of information.

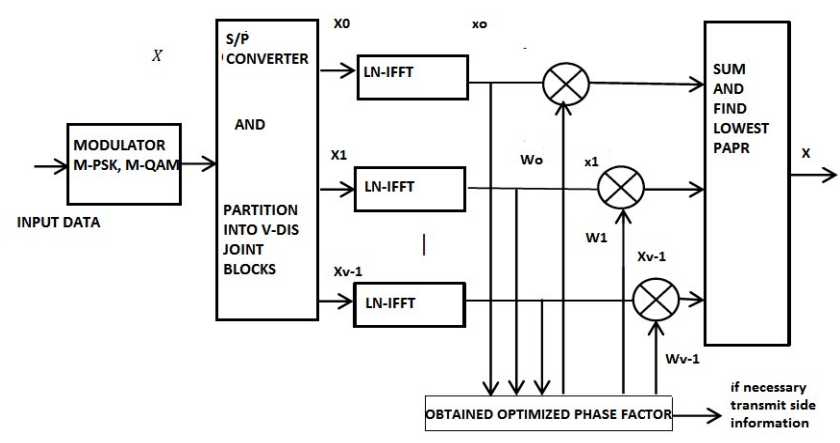

Figure 5. block diagram of Partial Transmit sequence

iii. Interleaved OFDM: The method is like a alternate of SLM, just the difference is that it uses an inter-leaver instead of using the sequences of phases. Interleaver is a computational device which has specific manner permutation and operates on N-symbols block. The interleaved OFDM's block diagram is as shown in Figure 6: Inter-leavers and de-inter-leavers are usually denoted by the symbol $\pi$ and $\pi^{-1}$.

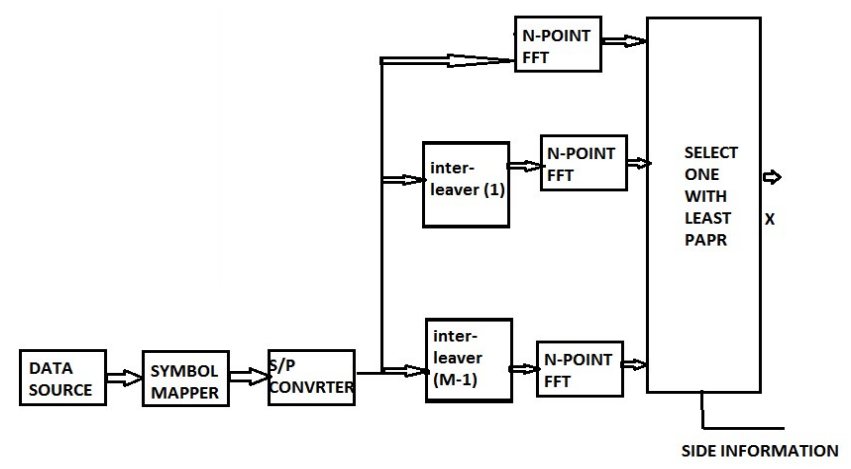

Figure 6. Block diagram of Interleaved OFDM

b) Signal distortion techniques: There are different types of that comes under signal distortion technique. The concept behind theses technique is that it distorts the original OFDM signal non linearity.

1. Clipping and filtering: One of the simplest approaches for reduction of PAPR of OFDM signal is clipping, is listed under the classification if signal distortion techniques. The technique uses a predefined threshold value

$$
x_{c}[n]=\left\{\begin{array}{ll}
-\mathrm{A} & \mathrm{x}[\mathrm{n}] \leq-\mathrm{A} \\
\mathrm{x}[\mathrm{n}] & |\mathrm{x}[\mathrm{n}]|<\mathrm{A} \\
\mathrm{A} & \mathrm{x}[\mathrm{n}]>\mathrm{A}
\end{array}\right\}
$$

Where $\mathrm{A}=$ pre-defined threshold value. $\mathrm{x}[\mathrm{n}]=$ pass band OFDM signal.

$\mathrm{x}_{\mathrm{c}}[\mathrm{n}]$-clipped version of $\mathrm{x}[\mathrm{n}]$.

With reference to the threshold it clips the amplitude peaks of the original OFDM signal. There may be reduction in PAPR of signal but there is chance of losing original information associated with the signal. Also it introduces in band distortion and out of band radiation using $_{\text {in }}$ filtering we may able to avoid the out of band radiation but filtering causes re-generation of peaks.

2. Tone reservation: It is an reservation method to reduce the PAPR. And this method uses some reserved tones and also some carriers are needed to be reserved in order to remove the PAPR. The selected subcarriers should be capable of reducing the PAPR of OFDM frame size. The complexity associated with this method is that it should have large number of reserved tones in order to reduce PAPR efficiently otherwise reduction in PAPR represents non negligible samples of available bandwidth. The merit of tone reservation technique is that is needs no processing and side information at the receiver.

3. Tone injection: with the use of this method the PAPR of multicarrier signal can be reduced efficiently without loss of data rate. In order to minimize the PAPR this method uses a 
set of equivalent constellation points which are being used by tone injection approach for original constellation points. The technique suffers from increased complexity due to requirement of additional IFFT operation; it also needs additional side information at the receiver for decoding process.

4. Peak windowing: unlike the method of clipping, peak windowing also uses a predetermined threshold value along with it is multiplied by weighing functions which are known as window functions. Most commonly used window function is Kaiser, Hamming, Henning Gaussian and cosine etc. since the scheme does. not employ hard clipping on the peaks of the signal it gives better performance result in reduction of PAPR as compared to the clipping technique But this technique cannot removes the distortion completely

5. Companding transform: this technique is a combination of compression and expansion which is basically applicable for audio signals. The principle behind this technique is that after companding lower peak values are increased but higher remains constant hence the peak of OFDM is increased resultantly the PAPR reduces. The drawback of the technique is that the average power needs to be maximized to decrease the PAPR which puts more burdens on transmitter relative with the average power.

\section{CODING TECHNIQUES}

The basic idea behind this technique is that it uses set of codes to reduce the PAPR. The code words are used before the application of IFFT. "When $\mathrm{N}$ signal are added with same phase they produce peak power which is $\mathrm{N}$ times greater than the average power". This doesn't produce any out band radiations and creates Do distortion is in OFDM signal but due the reason it uses set of code words the bandwidth efficiency is lesser when code rate is reduced. Complexity with this type of technique is that it needs to find best and suitable code words for reduction of OFDM and need to maintain the look up tables for coding and decoding.

\section{HYBRID PAPR REDUCTION TECHNIQUE}

The combination of SLM and pre-coding is used for reduction of PAPR.

1. Block Coding or pre-coding: The basic concept behind the scheme is the message symbols are chosen such a way that, the peak power of chosen symbols must be lesser. In pre-coding method, modulated data is multiplied with shaping matrix before the OFDM symbol formation the positive feature of the frequency selective multipath channel of OFDM system is utilized in pre-coding. In the processing initially the input data is modulated in baseband using modulation scheme such as M-PSK, M-QAM etc. precoding matrix transforms the baseband-modulated data stream. Different methods like pulse shaping function, discrete cosine transformation (DCT) matrix, Hadamard matrix, zadoff-chu sequence, generalized chirplike (GCL) sequence etc. are used to generate pre-coding matrix. There
After pre-coded data is transmitted through IFFT block to generate OFDM symbols. Each element of pre-coding matrix is carefully designed in such a way that it should reduce the PAPR. Since, here the modulated data is multiplied with pre-defined pre-coding matrix; there is no need of handshake between transmitter and receiver.

The fundamental concept behind the scheme is to use of two methodologies in combination. One is the "DCT matrix transform technique" and the other one is existing SLM technique. The transmitter block is showed in Fig 7.

We can consider this scheme as scheme 1 . Here at 0transmit end, the data stream is first applied to DCT matrix computation, and then the computed data is allowed process using SLM unit. The reduced autocorrelation coefficients of IFFT input reduces the OFDM signal PARP, which can be achieved by using DCT matrix before the application of IFFT.

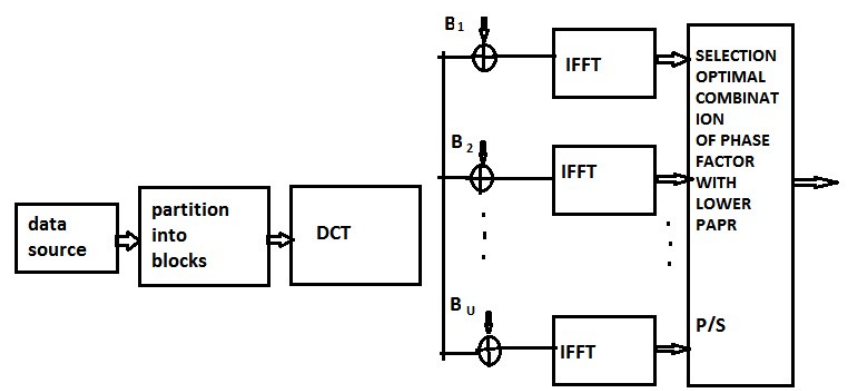

Figure 7. Diagram of proposed SLM scheme1

Again the DCT matrix is used after the processing of SLM unit further to minimize the PAPR value of signal. This procedure is considered as scheme 2 . In this way, processed by SLM processes the autocorrelation of signal is reduced by DCT matrix transform. The PAPR of fine output signal is further reduced. Fig. 8 describes the block diagram of Transmitter.

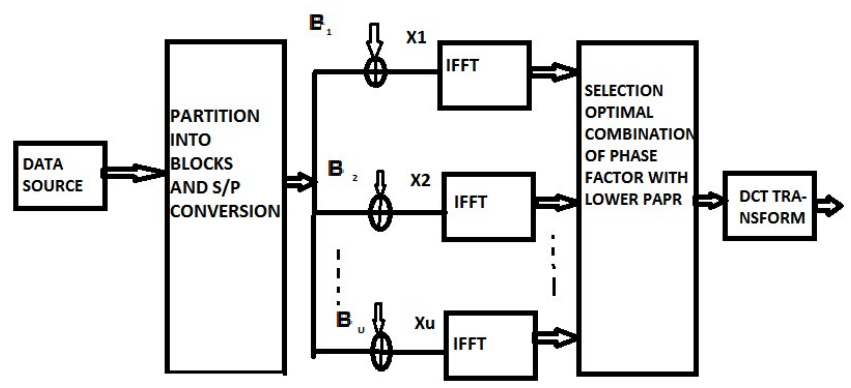

Figure 8. Diagram of proposed SLM scheme1

In order to minimize the high peak generation in signal, as compared to the Simplified SLM. The concept of Hadamard Transform is introduced which reduces the autocorrelation of the input sequence that reduces the peak to average power problem and no side information is needed at the receiver. The Hadamard SLM scheme is as depicted in Fig.9 
The following section givens we briefly review of Hadamard Transform. Assume that $\mathrm{H}$ is the Hadamard Transform matrix of orders N. Hadamard matrix is perfect orthogonal matrix. Each element of Hadamard matrix only is 1 or -1 . The Hadamard matrix of second order and is defined by $\mathrm{Y}=\mathrm{HX}$

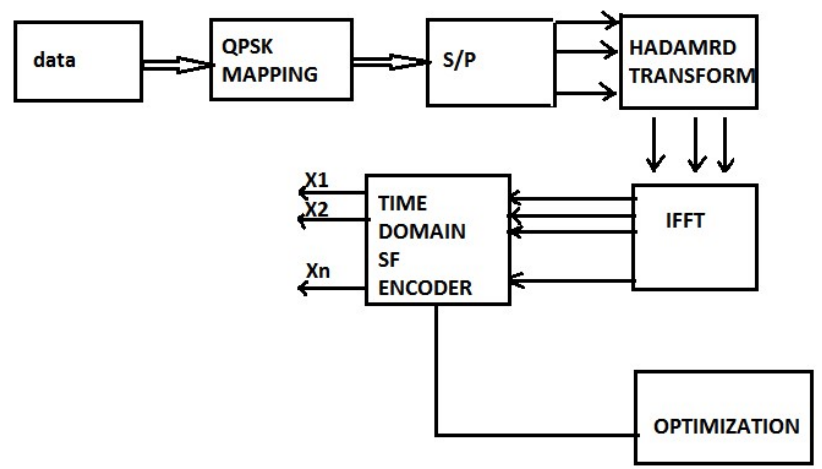

Figure 9. Block diagram of Hadamard SLM

$$
H 2=\frac{1}{\sqrt{2}}\left(\begin{array}{cc}
1 & 1 \\
1 & -1
\end{array}\right)
$$

The concept behind this Hadamard transform is that firstly Hadamard transform is applied to the coming input data stream there after the transformed data stream is presented as input to IFFT computation block. The signal processing step is below:

1. Hadamard matrix is applied to sequence $X$, i.e. $Y=H X$

2. Computation of Inverse Fourier transform i.e. IFFT $\mathrm{y}=\operatorname{IFFT}(\mathrm{Y})$

3. Equivalently candidate signal is generated in the time domain is applied to encoding operations by selecting least Maximum PAPR for transmission.

V. CDF \& CCDF OF PAPR: Cumulative distributed function $(\mathrm{CDF})$ is one of the parameter useful for measuring any of the PAPR techniques performance efficiency. The CDF of the signal can be mathematically defined as

$$
F(z)=1-\exp (z)
$$

And the complementary cumulative distributed function (CCDF) is alternate of CDF which measures the probability used instead of CDF which helps us to measure the probability of exceeding the given threshold by PAPR of few blocks.

$$
\begin{gathered}
\mathbf{P}(\mathbf{P A P R}>\mathrm{z})=1-\mathbf{P}(\mathbf{P A P R} \leq \mathrm{z}) \\
=1-\mathbf{F}(\mathrm{z}) \mathbf{N} \\
=1-(1-\exp (-\mathrm{z})) \mathrm{N}
\end{gathered}
$$

In the study pre-coder will be combined with scrambling technique and the system performance will be compared. SLM, PTS, DCT has got good performance in minimizing PAPR, along with this it increases computational complexity. There are many techniques several techniques based on low complexity SLM techniques. SLM side information index needs to transmit for each data block. SII is very much sensitive to system's error performance. Great security needs to be provided to these SII automatically leads to increase in complexity of the system in the discussion, a VLM pre-coded SLM and combination of DCT with SLM and HADAMARD with SLM techniques introduced to minimize the PAPR. Combination of precoding and scrambling techniques reduce PAPR much more as compare to other techniques

\section{CONCLUSION}

In the study, a survey on PAPR reduction we have discussed various techniques in categorization of "signal scrambling techniques" such as SLM, PTS and Interleaved OFDM have been discussed. Which are capable and reducing the OFDM efficiently but struck with system implementation complexity they need a handshake of signal between transmitter and receiver. The signal distortion technique studied such as peak windowing, clipping and filtering, tone reservation, tone injection companding transform are distort the original signal before the reception which may cause to loss of original information in OFDM signal also there might be creation of inbound distortion and out of band radiation. And the coding techniques needs an look up table to be maintained for decoding and encoding and they need of additional block code words to be added with the sequence of signal so feels to be better techniques does not produces 'in band distortion and out of band radiation' but provide no guarantee for spectral efficiency, and the new hybrid approach may provide better result in PAPR reduction with the integrated advantages if SLM and precoding. So finally with this study it is concluded that any individual PAPR reduction technique may not provide us the entire requirement that to be achieved but depending upon the need and requirement we can use the convenient technique for PAPR reduction. As per the study a signal scrambling technique like PTS may provide efficient result as compared to all other techniques also the hybrid peak reduction techniques are up to the few requirements so in future one can try use more hybrid combination technique in coordination with scrambling technique that gives the performance requirements.

\section{REFERENCES}

[1].A comparative survey on PAPR reduction techniques for ofdm performance improvement Volume 6, Issue 10, Oct 2015, pp. 53-65, Article ID: IJECET_06_10_006.

[2]. A Survey on Various PAPR Reduction Techniques in OFDM Communication Systems Volume: 02 Issue: 06 | Sep-2015.

[3]. Analysis of PAPR Reduction Schemes in LTEOFDMSystemIEEE International Conference on Advanced Research in Engineering and Technology (ICARET 2013).

[4]. Various Techniques to Reduce PAPR in OFDM Systems: A SurveyVol.8, No.11 (2015). 
[5]. PAPR reduction of ofdm signals using selective mapping and clipping Hybrid scheme bucharest, romania, august $27-31,2012$

[6]. Hybrid slm-pts for papr reduction in Mimo-ofdm Vol. 3, issue 5, may 2014

[7]. Y.-C.Wang and Z.-Q. Luo, "Optimized iterative clipping and filtering for papr reduction of ofdm signals," IEEE Transactions on Communications, vol. 59, no. 1, 2011.

[8].PAPR reduction of ofdm signals using selective mapping and clipping

Hybrid scheme 20th european signal processing conference (eusipco 2012) bucharest, romania, august 27 - 31, 2012. 